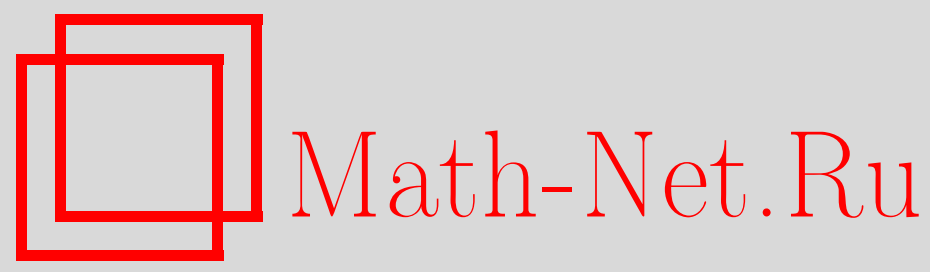

М. В. Балашов, Об аналоге теоремы Крейна-Мильмана для сильно выпуклой оболочки в гильбертовом пространстве, Матем. заметки, 2002, том 71, выпуск 1, 37-42

DOI: https://doi.org/10.4213/mzm326

Использование Общероссийского математического портала Math-Net.Ru подразумевает, что вы прочитали и согласны с пользовательским соглашением http://www.mathnet.ru/rus/agreement

Параметры загрузки:

IP : 54.162 .85 .209

26 апреля 2023 г., 15:32:17

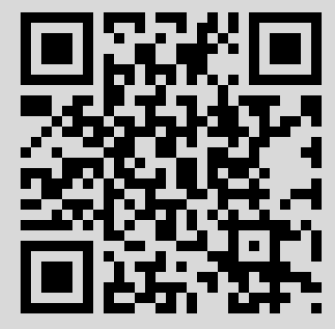




\section{ОБ АНАЛОГЕ ТЕОРЕМЫ КРЕЙНА-МИЛЬМАНА ДЛЯ СИЛЬНО ВЫПУКЛОЙ ОБОЛОЧКИ В ГИЛЬБЕРТОВОМ ПРОСТРАНСТВЕ}

\section{М.В. Балашов}

Доказана теорема: в гильбертовом пространстве замкнутое ограниченное множество содержится в сильно выпуклой $R$-оболочке своих $R$-сильно крайних точек. $R$-сильно крайние точки являются подмножеством крайних точек (и эти два множества могут не совпадать), сильно выпуклая $R$-оболочка множества содержит замыкание выпуклой оболочки множества.

Библиограффия: 5 названий.

1. Введение. Пусть $\mathscr{H}$ - гильбертово пространство над вещественным полем скаляров. Через $[x, y]$ будем обозначать отрезок с концами в точках $x$ и $y$. Через $B_{r}(a)$ обозначим шар радиуса $r$ с центром в точке $a$, т.е. $B_{r}(a)=\{x \in \mathscr{H} \mid\|x-a\| \leqslant r\}$, $\partial B_{r}(a)=\{x \in \mathscr{H} \mid\|x-a\|=r\}$. Через $\langle a, b\rangle$ обозначим скалярное произведение векторов $a, b \in \mathscr{H}$. Для множества $A \subset \mathscr{H}$ и точки $x$ введем $\rho(x, A)=\inf _{y \in A}\|x-y\|$. Через $P_{A}$ обозначим проектор на выпуклое замкнутое множество $A$. Расстояние в метрике Хаусдорфа между множествами $A$ и $B$ определяется по формуле

$$
h(A, B)=\inf \left\{r>0 \mid A \subset B+B_{r}(0), \quad B \subset A+B_{r}(0)\right\} .
$$

Введем также полезные обозначения: для $q \in \partial B_{1}(0), a \in \mathscr{H}$

$$
l(a, q)=\{a+\lambda q \mid \lambda \geqslant 0\}
$$

и

$$
\partial B_{r}(a, q)=\{x \in \mathscr{H} \mid\|x-a\|=r,\langle q, x-a\rangle \geqslant 0\},
$$

т.е. это "верхняя" (в смысле направления $q$ ) полусфера сферы $\partial B_{r}(a)$.

Сформулируем теперь определения и известные результаты.

ОПРедЕЛЕние 1.1 [1], [2]. Для множества $A \subset \mathscr{H}$ сильно выпуклой $R$-оболочкой называется множество, образованное пересечением всех шаров радиуса $R$, содержащих $A$. Обозначим это множество через $\operatorname{strco}_{R} A$.

Работа выполнена при поддержке Российского фонда фундаментальных исследований, грант № 98-01-00645, и Конкурсного центра фундаментального естествознания, грант № 97-0-1.9-26. 
ОПреДЕЛЕНИЕ $1.2[1],[2]$. Для множества $A \subset \mathscr{H}$ точка $x \in A$ назьвается $R$-сильно крайней, если для любьх двух точек $y, z \in A: y \neq x, z \neq x$, вьполнено $x \notin \operatorname{strco}_{R}\{y, z\}$. Будем обозначать множество $R$-сильно крайних точек $A$ через $\operatorname{extr}_{R} A$.

ОПреДЕЛЕНИЕ 1.3 [2]. Для множества $A \subset \mathscr{H}$ точка $x \in A$ назьвается $R$-выстyпающей, если существует шар радиуса $R$ с центром в точке $z$ такой, что $A \subset B_{R}(z)$ и $A \cap \partial B_{R}(z)=\{x\}$. Будем обозначать множество $R$-выступающих точек $A$ через $\exp _{R} A$.

Подмножество крайних точек множества $A$ обозначим через $\operatorname{extr} A$. Отметим, что если $\operatorname{strco}_{R} A \cap \operatorname{extr}_{R} A \neq \varnothing$, то $\operatorname{extr}_{R} A \subset \operatorname{extr} A$ и $\overline{\operatorname{co}} A \subset \operatorname{strco}_{R} A$.

В работе [2] была доказана следующая теорема.

Теорема 1.1. Пусть $A \subset \mathscr{H}$ - компакт в сильной топологии, $A$ содержится в uаре $B_{r}(a)$ u $r<R$. Tогдa $A \subset \operatorname{strco}_{R} \operatorname{extr}_{R} A$.

Целью настоящей работы является обобщение теоремы 1.1 на случай, когда множество $A$ - произвольное замкнутое множество, лежащее в шаре радиуса $r$.

Отличие приведенных в статье теорем 2.1 и 2.2 от известных обобщений теоремы Крейна-Мильмана для пространств с выпуклостью (см., например, теорему 4.7 из [3, c. 48]) заключается в том, что от множества мы не требуем ни компактности, ни выпуклости (которая, по сути, обеспечивает компактность множества в какой-либо более слабой топологии).

Отметим, что обобщение классической теоремы Крейна-Мильмана на случай замкнутых ограниченных множеств в банаховых пространствах, имеющих гладкое сопряженное (см. определение 1 из [4, гл. $5, \S 6]$ ), содержится в предложениях 3 и 4 из [4, гл. 5 , $\S 7]$.

\section{2. Аналог теоремы Крейна-Мильмана.}

Лемма 2.1. Пусть $A \subset \mathscr{H}$ - замкнутое в сильной топологии ограниченное множество. Тогда для всюду плотного мнохества $\mathscr{A} \subset \mathscr{H}$ (более того, $\mathscr{A}$ есть $G_{\delta}$-множество, т.е. пересечение не более чем счетного мнохества открытых всюду плотных мнохсеств) задача $\sup _{y \in A}\|x-y\|, x \in \mathscr{A}$, имеет единственное решение.

Доказательство этого важного для нас утверждения содержится в приложении II $\S 2$ раздела 2.1 из [5].

Докажем с помощью леммы 2.1 следующую лемму.

Лемма 2.2. Пусть $A \subset \mathscr{H}$ - замкнутое мнохсество, $A \subset B_{r}\left(z_{0}\right), r<R$. Тогда $\exp _{R} A \neq \varnothing$.

ДокАЗАТЕльСтво. Фиксируем $q \in \partial B_{1}(0)$. Пусть

$$
\lambda_{q}=\sup \left\{\lambda \geqslant 0 \mid A \subset B_{r}\left(z_{0}-\lambda q\right)\right\},
$$

и пусть $z_{q}=z_{0}-\lambda_{q} q$. Легко видеть, что $A \subset B_{r}\left(z_{q}\right)$.

Далее для простоты положим $z_{q}=0$. 
Фиксируем $\varepsilon \in(0, \min \{1, r / 2\})$ такое, что вьполнены следующие ограничения:

$$
\begin{gathered}
B_{\varepsilon^{4}}(-\varepsilon q) \subset B_{r}(0), \\
2 \varepsilon^{2} \sqrt{r^{2}+\varepsilon^{2}}+\varepsilon^{3}-\varepsilon^{6} \leqslant 1, \\
r+\varepsilon+\varepsilon^{4}<R .
\end{gathered}
$$

По лемме 2.1 существует точка $z_{\varepsilon} \in B_{\varepsilon^{4}}(-\varepsilon q)$ такая, что существует единственная точка $a \in A$, для которой

$$
\left\|z_{\varepsilon}-a\right\|=\sup _{x \in A}\left\|z_{\varepsilon}-x\right\|=r_{\varepsilon} .
$$

Таким образом, $\partial B_{r_{\varepsilon}}\left(z_{\varepsilon}\right) \cap A=\{a\}$. Отметим, что

$$
\left\|z_{\varepsilon}\right\| \leqslant \varepsilon+\varepsilon^{4}
$$

Покажем, что $a \in \exp _{R} A \cap \partial B_{r_{\varepsilon}}\left(z_{\varepsilon}, q\right)$. Это завершит доказательство.

Фиксируем произвольную точку $\xi \in B_{\varepsilon^{4}}(-\varepsilon q)$. Легко видеть, что в силу построения шара $B_{r}\left(z_{q}\right) \quad\left(z_{q}=0\right)$ найдется последовательность $a_{k} \in A$ и точки $x_{k} \in \partial B_{r}(0, q)$ такие, что $\left\|a_{k}\right\| \rightarrow r, \alpha_{k}=\left\|x_{k}-a_{k}\right\| \rightarrow 0$. Отсюда

$$
\sup _{x \in A}\|\xi-x\| \geqslant\left\|\xi-a_{k}\right\| \geqslant\left\|\xi-x_{k}\right\|-\alpha_{k} \geqslant \rho\left(\xi, \partial B_{r}(0, q)\right)-\alpha_{k},
$$

т.e.

$$
\begin{aligned}
\sup _{x \in A}\|\xi-x\| & \geqslant \rho\left(\xi, \partial B_{r}(0, q)\right) \\
& \geqslant \rho\left(-\varepsilon q, \partial B_{r}(0, q)\right)-\varepsilon^{4}=\sqrt{r^{2}+\varepsilon^{2}}-\varepsilon^{4} .
\end{aligned}
$$

С другой стороны,

$$
\sup _{x \in A}\|\xi-x\| \leqslant \sup _{x \in B_{r}(0)}\|\xi-x\| \leqslant r+\varepsilon+\varepsilon^{4}
$$

Из формул (2.2) и (2.3) получаем

$$
\sqrt{r^{2}+\varepsilon^{2}}-\varepsilon^{4} \leqslant r_{\varepsilon} \leqslant r+\varepsilon+\varepsilon^{4}
$$

Для векторов $-q$ и $x \in \mathscr{H}, x \neq 0$, введем угол $\varphi=\varphi(x)$ между ними; при этом $\cos \varphi=$ $\langle-q, x\rangle /\|x\|$. Пусть

$$
\begin{aligned}
& g(r, \varepsilon)=\frac{2 \varepsilon^{3} \sqrt{r^{2}+\varepsilon^{2}}-\varepsilon^{7}}{r}, \quad \varphi_{0}=\arccos g(r, \varepsilon), \\
& K=\left\{x \in \mathscr{H} \mid\left\langle-q, \frac{x}{\|x\|}\right\rangle \geqslant \cos \varphi_{0} \quad \text { или } x=0\right\},
\end{aligned}
$$

где $K$ есть острьй конус, симметричньй относительно прямой $\operatorname{lin}[q]$. 
Отметим, что в силу выбора $\varphi_{0}$ для всех $\varphi \in\left[0, \varphi_{0}\right] \cos \varphi \geqslant g(r, \varepsilon)$, т.е.

$$
\sqrt{r^{2}+\varepsilon^{2}-2 r \varepsilon \cos \varphi}+\varepsilon^{4}<\sqrt{r^{2}+\varepsilon^{2}}-\varepsilon^{4}
$$

Допустим, что $a \in B_{r}(0) \cap K$. Тогда для $\xi=z_{\varepsilon}$ по формуле (2.2) имеем

$$
\left\|z_{\varepsilon}-a\right\| \geqslant \rho\left(z_{\varepsilon}, \partial B_{r}(0, q)\right)
$$

$\mathrm{C}$ другой стороны, $\varphi(a) \in\left[0, \varphi_{0}\right]$ и в силу ограничений на $\varepsilon(O 1)-(O 3)$ справедливы оценки

$$
\begin{aligned}
\left\|z_{\varepsilon}-a\right\| & \leqslant\|a+\varepsilon q\|+\varepsilon^{4}=\sqrt{\|a\|^{2}+\varepsilon^{2}-2\|a\| \varepsilon \cos \varphi(a)}+\varepsilon^{4} \\
& \leqslant \sqrt{r^{2}+\varepsilon^{2}-2 r \varepsilon \cos \varphi(a)}+\varepsilon^{4}<\sqrt{r^{2}+\varepsilon^{2}}-\varepsilon^{4} \leqslant \rho\left(z_{\varepsilon}, \partial B_{r}(0, q)\right)
\end{aligned}
$$

(заключительное неравенство из $(2.2)$ с $\xi=z_{\varepsilon}$ ). Последние две формулы противоречат друг другу, поэтому $a \in B_{r}(0) \backslash K$.

Покажем, что

$$
B_{r}(0) \backslash K \subset\left\{x \in \mathscr{H} \mid\left\langle q, x+\left(\varepsilon-\varepsilon^{4}\right) q\right\rangle \geqslant 0\right\}=\Pi_{q}
$$

Пусть $x \in B_{r}(0) \backslash K, x \neq 0$. Тогда $r x /\|x\| \in B_{r}(0) \backslash K$; поэтому без ограничения общности $\|x\|=r$.

Соотношение $x \notin K$ влечет два случая.

a) $x \in \partial B_{r}(0, q)$ и тогда включение $x \in \Pi_{q}$ очевидно.

б) $x \in \partial B_{r}(0,-q)$ и угол между векторами $x$ и $-q$ больше $\varphi_{0}$.

Тогда $(1 / r)\langle x,-q\rangle \leqslant \cos \varphi_{0}=g(r, \varepsilon)$,

$$
\langle x,-q\rangle \leqslant r g(r, \varepsilon) \leqslant\left(\varepsilon-\varepsilon^{4}\right)\langle q, q\rangle
$$

(последнее неравенство верно в силу $(O 2)$ ). Отсюда

$$
\left\langle q, x+q\left(\varepsilon-\varepsilon^{4}\right)\right\rangle \geqslant 0,
$$

т.e. $x \in \Pi_{q}$.

Включение $a \in \partial B_{r_{\varepsilon}}\left(z_{\varepsilon}\right)$ вместе с включением

$$
\Pi_{q} \subset\left\{x \in \mathscr{H} \mid\left\langle q, x-z_{\varepsilon}\right\rangle \geqslant 0\right\}
$$

влечет включение $a \in \partial B_{r_{\varepsilon}}\left(z_{\varepsilon}, q\right)$. В силу $(O 3)$ и (2.4) $r_{\varepsilon}<R$. Пусть $p=\left(a-z_{\varepsilon}\right) /\left\|a-z_{\varepsilon}\right\|$. Тогда

$$
A \subset B_{r_{\varepsilon}}\left(z_{\varepsilon}\right) \subset B_{R}(a-R p), \quad \partial B_{r_{\varepsilon}}\left(z_{\varepsilon}\right) \cap \partial B_{R}(a-R p)=\{a\},
$$

т.е. по определению $a \in \exp _{R} A \cap \partial B_{r_{\varepsilon}}\left(z_{\varepsilon}, q\right)$. 
Теорема 2.1. Пусть $A \subset \mathscr{H}$ замкнутое в сильной топологии множество, $A \subset B_{\rho}\left(z_{0}\right), \rho<R$. Тогда

$$
A \subset \operatorname{strco}_{R} \exp _{R} A \text {. }
$$

ДокАЗАтЕЛЬСтво. Пусть $B=\operatorname{strco}_{R} \exp _{R} A$. Допустим, что существует $x \in A \backslash B$. В силу определения 1.1 найдется такой шар $B_{R}(z)$, что $B \subset B_{R}(z)$ и $x \notin B_{R}(z)$. Пусть $y=P_{B_{R}(z)} x, \varepsilon=\|x-y\|>0$.

Определим шар $B_{r}\left(z_{0}\right)$, где $r>0$ удовлетворяет условию

$$
r=\max \left\{\rho, \frac{R+\varepsilon+\sqrt{(R-\varepsilon)^{2}-\varepsilon^{2}}}{2}\right\} \quad \text { при } R \geqslant 2 \varepsilon, r=\rho \text { при } R<2 \varepsilon .
$$

Отметим, что $\rho \leqslant r<R$ и $z \neq z_{0}$.

1. Пусть $q=\left(z_{0}-z\right) /\left\|z_{0}-z\right\|$. Введем

$$
\begin{gathered}
a=z_{0}+\left\|z_{0}-x\right\| q \in B_{r}\left(z_{0}\right) \\
\|a-z\|=\left\|z-z_{0}\right\|+\left\|x-z_{0}\right\| \geqslant\|x-z\|>R
\end{gathered}
$$

откуда $a \notin B_{R}(z)$. По свойству проекции $P_{B_{R}(z)} a=z+R q$; следовательно,

$$
P_{B_{R}(z)} l(z+R q, q)=\{z+R q\} \text {. }
$$

Так как $\|x-y\|=\|x-z\|-R$ и $\|a-(z+q R)\|=\|a-z\|-R$, то с учетом $(2.6)$

$$
\|a-(z+R q)\|=\|a-z\|+\|x-y\|-\|x-z\| \geqslant\|x-y\|=\varepsilon .
$$

Так как $B \subset B_{R}(z) \cap B_{r}\left(z_{0}\right)$ и $x \in B_{r}\left(z_{0}\right) \backslash B_{R}(z)$, то $\left\|z-z_{0}\right\| \in(R-r, R+r)$, откуда с учетом определения $q\left\|z-z_{0}+R q\right\|=\left|R-\left\|z-z_{0}\right\|\right| \leqslant r$,

$$
z+R q \in B_{r}\left(z_{0}\right)
$$

Из формул $(2.5),(2.7),(2.8)$ и (2.9) следует, что для любого шара $B_{r}\left(z_{1}\right)$ такого, что $A \subset B_{r}\left(z_{1}\right)$ и $z_{1} \in l(z, q)$, отрезок $[z+R q, z+(R+\varepsilon) q]$ лежит в $B_{r}\left(z_{1}\right)$.

2. По лемме 2.2 найдется максимальное $\lambda_{q} \geqslant 0: A \subset B_{r}\left(z_{0}-\lambda_{q} q\right)$. Пусть без ограничения общности $z_{0}-\lambda_{q} q=0$.

В силу пункта 1 доказательства найдется $\delta \geqslant \varepsilon$, для которого $[z+R q, z+(R+\delta) q] \subset$ $B_{r}(0)$ и $z+(R+\delta) q \in \partial B_{r}(0)$. Так как $q \in \operatorname{lin}\left[z_{0}-z\right]$, то $z+(R+\delta) q=r q$ и $z=-(R-r+\delta) q$.

Фиксируем произвольное $u \in \partial B_{r}(0, q)$, т.е. $\|u\|=r$ и $\langle u, q\rangle \geqslant 0$. Тогда

$$
\|u-z\|^{2}-R^{2} \geqslant 2 r^{2}-2 r(R+\varepsilon)+2 \varepsilon R+\varepsilon^{2}>0,
$$

откуда

$$
\exists \alpha>0 \quad \forall u \in \partial B_{r}(0, q) \quad \rho\left(u, B_{R}(z)\right) \geqslant \alpha .
$$

По лемме 2.2 для любого $\varepsilon>0$ найдется шар $B_{r_{\varepsilon}}\left(z_{\varepsilon}\right)$ (в обозначениях леммы 2.2) такой, что $A \subset B_{r_{\varepsilon}}\left(z_{\varepsilon}\right)$ и $\partial B_{r_{\varepsilon}}\left(z_{\varepsilon}\right) \cap A=\partial B_{r_{\varepsilon}}\left(z_{\varepsilon}, q\right) \cap A=\{a\}$. Выбирая малые $\varepsilon>0$, 
удовлетворяющие $(O 1)-(O 3)$, можно удовлетворить условиям $r_{\varepsilon} \in[r-\alpha / 4, r+\alpha / 4]$, $\left\|z_{\varepsilon}\right\| \leqslant \alpha / 4$ (см. $\left.(2.1),(2.4)\right)$. Фиксируем такое $\varepsilon$ и соответствующие ему $z_{\varepsilon}, r_{\varepsilon}$. Имеем

$$
\begin{aligned}
& h\left(\partial B_{r}(0, q), \partial B_{r_{\varepsilon}}\left(z_{\varepsilon}, q\right)\right) \leqslant h\left(\partial B_{r}(0, q), \partial B_{r_{\varepsilon}}(0, q)\right) \\
& \quad+h\left(\partial B_{r_{\varepsilon}}(0, q), \partial B_{r_{\varepsilon}}\left(z_{\varepsilon}, q\right)\right) \leqslant\left|r-r_{\varepsilon}\right|+\left\|z_{\varepsilon}\right\| \leqslant \frac{\alpha}{2} .
\end{aligned}
$$

Отсюда с учетом условия (2.10)

$$
\partial B_{r_{\varepsilon}}\left(z_{\varepsilon}, q\right) \cap B_{R}(z)=\varnothing
$$

Из формулы (2.11) следует, что $a \notin B_{R}(z)$. Поскольку $r_{\varepsilon}<R$ (см. (O3) и (2.4)), то, повторяя рассуждения леммы 2.1, $a \in \exp _{R} A$. Этопротиворечит включению $\exp _{R} A \subset$ $B \subset B_{R}(z)$.

В работе [2] показано, что для любого множества $A \subset \mathscr{H} x \in \exp _{R} A \Rightarrow x \in \operatorname{extr}_{R} A$, отсюда и из теоремы 2.1 немедленно следует теорема.

ТеОрема 2.2. Пусть $A \subset \mathscr{H}$ замкнутое в сильной топологии множество, $A \subset B_{\rho}\left(z_{0}\right), \rho<R$. Тогда

$$
A \subset \operatorname{strco}_{R} \operatorname{extr}_{R} A
$$

\section{СПИСОК ЦИТИРОВАННОЙ ЛИТЕРАТУРЫ}

[1] Половинкин Е. С. Сильно выпуклый анализ // Матем. сб. 1996. Т. 187. № 2. С. 103-130.

[2] Балашов М. В., Половинкин Е. С. $M$-сильно выпуклые подмножества и их порождающие множества // Матем. сб. 2000. Т. 191. №1. С. 26-64.

[3] Солтан В. П. Введение в аксиоматическую теорию выпуклости. Кишинев: Штиинца, 1984.

[4] Обен ЖК.-П., Экланд И. Прикладной нелинейный анализ. М.: Мир, 1988.

[5] Экланд И., Темам Р. Выпукльй анализ и вариационные проблемы. М.: Мир, 1979. 